\title{
Thermodynamics of interacting entropy-corrected holographic dark energy in a non-flat FRW universe
}

\author{
Mubasher Jamil,,$* *$ Ahmad Sheykhi, ${ }^{2,+}$ and M. Umar Farooq ${ }^{1,+}$ \\ ${ }^{1}$ Center for Advanced Mathematics and Physics, \\ National University of Sciences and Technology, H-12, Islamabad, Pakistan \\ ${ }^{2}$ Department of Physics, Shahid Bahonar University, P.O. Box 76175, Kerman, Iran
}

\begin{abstract}
A so-called "entropy-corrected holographic dark energy" (ECHDE), was recently proposed to explain the dark energy-dominated universe with the help of quantum corrections to the entropy-area relation in the setup of loop quantum cosmology. Using this new definition, we investigate its thermodynamical features including entropy and energy conservation. We describe the thermodynamical interpretation of the interaction between ECHDE and dark matter in a non-flat universe. We obtain a relation between the interaction term of the dark components and thermal fluctuation. Our study further generalizes the earlier works [M.R. Setare and E.C. Vagenas, Phys. Lett. B 666 (2008) 111; B. Wang et al., Phys. Lett. B 662 (2008) 1] in this direction.
\end{abstract}

\footnotetext{
*Electronic address: mjamil@camp.nust.edu.pk

$\dagger$ Electronic address: sheykhi@mail.uk.ac.ir

${ }^{\ddagger}$ Electronic address: mfarooq@camp.nust.edu.pk
} 


\section{INTRODUCTION}

There is a wide consensus among cosmologists that our universe has entered a phase of accelerated expansion likely driven by some unknown energy component whose main feature is to possess a negative pressure [1]. Although the nature of such dark energy is still speculative, an overwhelming flood of papers has appeared which attempt to describe it by devising a great variety of models. Among them are cosmological constant, exotic fields such as phantom or quintessence, modified gravity, etc, see [2] for a recent review.

An interesting attempt for probing the nature of dark energy within the framework of quantum gravity, is the so-called "Holographic Dark Energy" (HDE) proposal. This model which has arisen a lot of enthusiasm recently [3 3 ], is motivated from the holographic hypothesis [10] and has been tested and constrained by various astronomical observations [11]. The definition of HDE is originally motivated from the entropy-area relation which depends on the theory of gravity under consideration. In the thermodynamics of black hole, there is a maximum entropy in a box of length $L$, commonly termed, the Bekenstein-Hawking entropy bound, $S \sim M_{p}^{2} L^{2}$, which scales as the area of the box $A \sim L^{2}$ rather than the volume $V \sim L^{3}$. Here $M_{p}^{2}=(8 \pi G)^{-1}$ is the reduced Planck mass and throughout this Paper we use the units $c=\hbar=1$. In this context, Cohen et al. [3] suggested that in quantum field theory a short distance cutoff is related to a long distance cutoff due to the limit set by formation of a black hole, which results in an upper bound on the zero-point energy density. In line with this suggestion, Hsu and Li [4, 5] argued that this energy density could be viewed as the holographic dark energy density satisfying $\rho_{\Lambda}=3 n^{2} M_{p}^{2} / L^{2}$, where $L$ is the size of a region which provides an IR cut-off, and the numerical constant $3 n^{2}$ is introduced for convenience. It is important to note that in the literature, various scenarios of HDE have been studied via considering different system's IR cutoff. In the absence of interaction between dark matter and dark energy in flat universe, Li [5] discussed three choices for the length scale $L$ which is supposed to provide an IR cutoff. The first choice is the Hubble radius, $L=H^{-1}[4]$, which leads to a wrong equation of state, namely that for dust. The second option is the particle horizon radius. In this case it is impossible to obtain an accelerated expansion. Only the third choice, the identification of $L$ with the radius of the future event horizon gives the desired result, namely a sufficiently negative equation of state to obtain an accelerated universe. However, as soon as an interaction between dark 
energy and dark matter is taken into account, the first choice, $L=H^{-1}$, in flat universe, can simultaneously drive accelerated expansion and solve the coincidence problem [12]. It was also demonstrated that in the presence of an interaction, in a non-flat universe, the natural choice for IR cutoff could be the apparent horizon radius [13].

As we mentioned the black hole entropy $S$ plays a crucial role in the derivation of HDE. Indeed, the definition and derivation of holographic energy density $\left(\rho_{\Lambda}=3 n^{2} M_{p}^{2} / L^{2}\right)$ depends on the entropy-area relationship $S \sim A \sim L^{2}$ of black holes in Einsteins gravity, where $A \sim L^{2}$ represents the area of the horizon. However, this definition can be modified from the inclusion of quantum effects, motivated from the loop quantum gravity (LQG). The quantum corrections provided to the entropy-area relationship leads to the curvature correction in the Einstein-Hilbert action and vice versa [14]. The corrected entropy takes the form [15]

$$
S=\frac{A}{4 G}+\gamma \ln \frac{A}{4 G}+\beta
$$

where $\gamma$ and $\beta$ are dimensionless constants of order unity. The exact values of these constants are not yet determined and still an open issue in loop quantum cosmology. These corrections arise in the black hole entropy in LQG due to thermal equilibrium fluctuations and quantum fluctuations [16]. Taking the corrected entropy-area relation (1) into account, the energy density of the HDE will be modified as well. On this basis, Wei [17] proposed the energy density of the so-called "entropy-corrected holographic dark energy" (ECHDE) in the form

$$
\rho_{\Lambda}=3 n^{2} M_{p}^{2} L^{-2}+\gamma L^{-4} \ln \left(M_{p}^{2} L^{2}\right)+\beta L^{-4} .
$$

In the special case $\gamma=\beta=0$, the above equation yields the well-known holographic energy density. Since the last two terms in Eq. (2) can be comparable to the first term only when $L$ is very small, the corrections make sense only at the early stage of the universe. When the universe becomes large, ECHDE reduces to the ordinary HDE.

In this Paper we intend to study thermodynamical interpretation of the interaction between dark matter and ECHDE for a universe with spacial curvature. As systems's IR cutoff we shall choose the radius of the event horizon measured on the sphere of the horizon, defined as $L=\operatorname{ar}(t)$. Using the logarithmic correction to the equilibrium entropy we will derive an expression for the interaction term in terms of a thermal fluctuation. This Paper is outlined as follows. In the next section we consider the thermodynamical picture of the non-interacting ECHDE in a non-flat universe. In section III, we extend the thermodynam- 
ical description in the case where there is an interaction term between the dark components. We also present an expression for the interaction term in terms of a thermal fluctuation in this section. The last section is devoted to conclusion.

\section{ENTROPY CORRECTED HDE IN A NON-FLAT UNIVERSE}

We assume the background spacetime to be spatially homogeneous and isotropic given by Friedmann-Robertson-Walker (FRW) metric

$$
d s^{2}=d t^{2}-a^{2}(t)\left[\frac{d r^{2}}{1-k r^{2}}+r^{2}\left(d \theta^{2}+\sin ^{2} \theta d \phi^{2}\right)\right],
$$

where $a(t)$ is the dimensionless scale factor which is an arbitrary function of time, and $k$ is the curvature parameter with $k=-1,0,1$ corresponding to open, flat, and closed universes, respectively. The Einstein field equation representing the dynamics of the FRW spacetime can be written as

$$
H^{2}+\frac{k}{a^{2}}=\frac{1}{3 M_{p}^{2}}\left(\rho_{\Lambda}+\rho_{m}\right),
$$

where $H=\dot{a} / a$ is the Hubble parameter and $\rho_{\Lambda}$ and $\rho_{m}$ are the energy densities of dark energy and dark matter, respectively. One can rewrite Eq. (4) in the dimensionless form as

$$
1+\Omega_{k}=\Omega_{\Lambda}+\Omega_{m}
$$

where the above density parameters are defined by

$$
\Omega_{m}=\frac{\rho_{m}}{\rho_{c r}}=\frac{\rho_{m}}{3 H^{2} M_{p}^{2}}, \quad \Omega_{\Lambda}=\frac{\rho_{\Lambda}}{\rho_{c r}}=\frac{\rho_{\Lambda}}{3 H^{2} M_{p}^{2}}, \quad \Omega_{k}=\frac{k}{(a H)^{2}} .
$$

Here $\rho_{c r}$ is the critical energy density. The energy conservation equations for dark energy and matter are

$$
\begin{aligned}
\dot{\rho}_{\Lambda}+3 H\left(1+w_{\Lambda}^{0}\right) \rho_{\Lambda} & =0, \\
\dot{\rho}_{m}+3 H \rho_{m} & =0,
\end{aligned}
$$

Notice that in the above equation, we have assumed matter to be pressure-less fluid, $w_{m}=0$. As we shall see later, this may not be the case if matter interacts with dark energy.

Due to deep insights of Bekenstein [18] and Hawking [19], there has been established a connection between gravity and thermodynamics. With the help of semi-classical analysis of 
static black holes with a vacuum background, Hawking proposed that black holes could not remain static but can lose mass by emitting virtual particles from their horizons. From the pure relativistic point of view, black holes are 'cold' objects without any temperature. But Hawking's analysis implied that black holes are not only 'hot' but can gradually increase their temperature with evaporation. Thus a black hole can explode if its mass is reduced closer to Planck's mass. The analysis showed that the black hole's horizon temperature is inversely proportional to its mass. Bekenstein [18] thought that since the entropy of the black hole horizon is proportional to horizon area, hence with evaporation, the black hole's entropy will decrease with time and violate the second law of thermodynamics. As a black hole loses entropy, the entropy of the background universe increases. Bekenstein suggested that the sum of black hole entropy and the background entropy must be an increasing quantity with respect to time. It was a matter of doubt whether the temperature associated with the horizon is a physical temperature or merely a geometrical effect. To answer this, Padmanabhan [20] proved that this temperature is a physical quantity like temperature of physical objects.

In this Paper, following [17] we assume the energy density of the HDE is modified due to the correction terms in the entropy formula. Hence the energy density of the ECHDE takes the form (2) where $L$ is defined as

$$
L=a(t) \frac{\sin (\sqrt{|k|} y)}{\sqrt{|k|}}, \quad y=\frac{R_{h}}{a(t)},
$$

where $R_{h}$ is the size of the future event horizon defined as

$$
R_{h}=a(t) \int_{t}^{\infty} \frac{d t^{\prime}}{a\left(t^{\prime}\right)}=a(t) \int_{0}^{r_{1}} \frac{d r}{\sqrt{1-k r^{2}}} .
$$

The last integral has the explicit form as

$$
\int_{0}^{r_{1}} \frac{d r}{\sqrt{1-k r^{2}}}=\frac{1}{\sqrt{|k|}} \sin ^{-1}\left(\sqrt{|k|} r_{1}\right)= \begin{cases}\sin ^{-1}\left(r_{1}\right), & k=+1, \\ r_{1}, & k=0, \\ \sinh ^{-1}\left(r_{1}\right), & k=-1,\end{cases}
$$

Using the definitions of $\Omega_{\Lambda}$ and $\rho_{c r}$, it is straightforward to show that

$$
H L=\sqrt{\frac{3 n^{2} M_{p}^{2}+\gamma L^{-2} \ln \left(M_{p}^{2} L^{2}\right)+\beta L^{-2}}{3 M_{p}^{2} \Omega_{\Lambda}^{0}}} .
$$


Differentiating $L$ with respect to time $t$ and using (12) yields

$$
\dot{L}=\sqrt{\frac{3 n^{2} M_{p}^{2}+\gamma L^{-2} \ln \left(M_{p}^{2} L^{2}\right)+\beta L^{-2}}{3 M_{p}^{2} \Omega_{\Lambda}^{0}}}-\cos (\sqrt{|k|} y),
$$

where

$$
\cos (\sqrt{|k|} y)= \begin{cases}\cos y & k=+1 \\ 1 & k=0 \\ \cosh y & k=-1\end{cases}
$$

Differentiating (2) with respect to $t$ gives

$$
\begin{aligned}
\dot{\rho}_{\Lambda}= & {\left[2 \gamma L^{-5}-4 \gamma L^{-5} \ln \left(M_{p}^{2} L^{2}\right)-4 \beta L^{-5}-6 n^{2} M_{p}^{2} L^{-3}\right] } \\
& \times\left[\sqrt{\frac{3 n^{2} M_{p}^{2}+\gamma L^{-2} \ln \left(M_{p}^{2} L^{2}\right)+\beta L^{-2}}{3 M_{p}^{2} \Omega_{\Lambda}^{0}}}-\cos (\sqrt{|k|} y)\right] .
\end{aligned}
$$

Making use of (15) in (7) gives

$$
\begin{aligned}
w_{\Lambda}^{0}= & -1-\left(\frac{2 \gamma L^{-2}-4 \gamma L^{-2} \ln \left(M_{p}^{2} L^{2}\right)-4 \beta L^{-2}-6 n^{2} M_{p}^{2}}{3\left(3 n^{2} M_{p}^{2}+\gamma L^{-2} \ln \left(M_{p}^{2} L^{2}\right)+\beta L^{-2}\right)}\right) \\
& \times\left[1-\sqrt{\frac{3 M_{p}^{2} \Omega_{\Lambda}^{0}}{3 n^{2} M_{p}^{2}+\gamma L^{-2} \ln \left(M_{p}^{2} L^{2}\right)+\beta L^{-2}}} \cos (\sqrt{|k|} y)\right] .
\end{aligned}
$$

The above expression represents the equation of state of the entropy corrected holographic dark energy.

After the discovery of black hole thermodynamics, people got interested in understanding whether one can associate thermodynamics to the cosmological horizons as well similar to black hole horizons [21]. There is a fundamental difference between cosmological and black hole event horizons: in the former case, the observer lies inside the horizon while in the later case; the observer is outside the horizon, hence the temperature measurement by both the observers may be different. Moreover, if the thermodynamics of horizons is restricted only to event horizons, then the later quantity does not always exist for the cosmological spacetimes. Cai and collaborators [22] have proposed that one can associate the Hawking temperature to the horizon of FRW cosmological spacetime if the universe is enclosed by an apparent horizon (a trapped surface with vanishing expansion) and not an event horizon. This temperature is inversely proportional to the size of the apparent horizon.

The FRW universe may contain several cosmic ingredients including dark energy, dark matter and radiation. Astrophysical observations suggest that the energy density of dark 
energy is the dominant quantity in the universe. In the present analysis, we shall assume the FRW universe to contain only dark energy and matter. Astrophysically, the temperature of dark energy and matter could be different from that of the apparent horizon: if the temperature of cosmic fluids is hotter than the apparent horizon then heat will flow outside the horizon and vice versa. It is possible that the physical system (consisting of dark energy, matter and the apparent horizon) ultimately reaches the state of thermal equilibrium in a finite time. Hence one can take the same temperature for the whole thermodynamic system. In this Paper, we shall assume the dark energy to be given by the entropy corrected holographic dark energy.

The first law of thermodynamics is defined as

$$
T d S_{\Lambda}=d E_{\Lambda}+p_{\Lambda} d V
$$

where volume $V$ is given as

$$
V=\frac{4 \pi}{3} L^{3}
$$

the energy of the holographic dark energy is defined as

$$
E_{\Lambda}=\rho_{\Lambda} V=\frac{4}{3} \pi\left(3 n^{2} M_{p}^{2} L+\gamma L^{-1} \ln \left(M_{p}^{2} L^{2}\right)+\beta L^{-1}\right),
$$

and the temperature of the event horizon is given as

$$
T=\frac{1}{2 \pi L^{0}} .
$$

Substituting the aforementioned expressions for the volume, energy and temperature in Eq. (17) for the case of the non-interacting ECHDE model, one obtains

$d S_{\Lambda}^{(0)}=\frac{8 \pi^{2}}{3}\left[3 n^{2} M_{p}^{2}\left(1+3 w_{\Lambda}^{0}\right)-\left(\gamma\left(L^{0}\right)^{-2} \ln \left(M_{p}^{2}\left(L^{0}\right)^{2}\right)+\beta\left(L^{0}\right)^{-2}\right)\left(1-3 w_{\Lambda}^{0}\right)+2 \gamma\left(L^{0}\right)^{-2}\right] L^{0} d L^{0}$,

where

$$
\begin{aligned}
& 1+3 \omega_{\Lambda}^{0}=-2-\frac{2 \gamma\left(L^{0}\right)^{-2}-4 \gamma\left(L^{0}\right)^{-2} \ln \left(M_{p}^{2}\left(L^{0}\right)^{2}\right)-4 \beta\left(L^{0}\right)^{-2}-6 n^{2} M_{p}^{2}}{3 n^{2} M_{p}^{2}+\gamma\left(L^{0}\right)^{-2} \ln \left(M_{p}^{2} L^{2}\right)+\beta\left(L^{0}\right)^{-2}} \\
& \times\left[1-\sqrt{\frac{3 M_{p}^{2} \Omega_{\Lambda}^{0}}{3 n^{2} M_{p}^{2}+\gamma\left(L^{0}\right)^{-2} \ln \left(M_{p}^{2} L^{2}\right)+\beta\left(L^{0}\right)^{-2}}} \cos (\sqrt{|k|} y)\right], \\
& 1-3 \omega_{\Lambda}^{0}=4+\frac{2 \gamma\left(L^{0}\right)^{-2}-4 \gamma\left(L^{0}\right)^{-2} \ln \left(M_{p}^{2}\left(L^{0}\right)^{2}\right)-4 \beta\left(L^{0}\right)^{-2}-6 n^{2} M_{p}^{2}}{3 n^{2} M_{p}^{2}+\gamma\left(L^{0}\right)^{-2} \ln \left(M_{p}^{2}\left(L^{0}\right)^{2}\right)+\beta\left(L^{0}\right)^{-2}} \\
& \times\left[1-\sqrt{\frac{3 M_{p}^{2} \Omega_{\Lambda}^{0}}{3 n^{2} M_{p}^{2}+\gamma\left(L^{0}\right)^{-2} \ln \left(M_{p}^{2}\left(L^{0}\right)^{2}\right)+\beta\left(L^{0}\right)^{-2}}} \cos (\sqrt{|k|} y)\right] .
\end{aligned}
$$




\section{THERMODYNAMICS OF INTERACTING ECHDE}

In this section we consider the interaction between ECHDE $\rho_{\Lambda}$ and matter $\rho_{m}$. Given the unknown nature of both dark energy and dark matter there is nothing in principle against their mutual interaction and it seems very special that these two major components in the universe are entirely independent. Indeed, this possibility has received a lot of attention recently [23-26] and in particular, it has been shown that the appropriate coupling between dark components can influence the perturbation dynamics and the cosmic microwave background (CMB) spectrum and account for the observed CMB low $l$ suppression [27]. It was shown that in a model with interaction the structure formation has a different fate as compared with the non-interacting case [27]. It was also discussed that with strong coupling between dark energy and dark matter, the matter density perturbation is stronger during the universe evolution till today, which shows that the interaction between dark energy and dark matter enhances the clustering of dark matter perturbation compared to the noninteracting case in the past. Therefore, the coupling between dark components could be a major issue to be confronted in studying the physics of dark energy. However, so long as the nature of these two components remain unknown it will not be possible to derive the precise form of the interaction from first principles. Therefore, one has to assume a specific

coupling from the outset [28, 29] or determine it from phenomenological requirements [30]. Thermodynamical description of the interaction (coupling) between holographic dark energy and dark matter has been studied in [31].

In the presence of interaction the corresponding conservation equations are written as

$$
\begin{aligned}
\dot{\rho}_{\Lambda}+3 H\left(1+w_{\Lambda}\right) \rho_{\Lambda} & =-Q \\
\dot{\rho}_{m}+3 H \rho_{m} & =Q
\end{aligned}
$$

The energy conservation equation (24) gives

$$
\begin{aligned}
1+3 \omega_{\Lambda}= & -2-\frac{Q}{3 M_{p}^{2} H^{3} \Omega_{\Lambda}}-\left(\frac{2 \gamma L^{-2}-4 \gamma L^{-2} \ln \left(M_{p}^{2} L^{2}\right)-4 \beta L^{-2}-6 n^{2} M_{p}^{2}}{3 n^{2} M_{p}^{2}+\gamma L^{-2} \ln \left(M_{p}^{2} L^{2}\right)+\beta L^{-2}}\right) \\
& \times\left[1-\sqrt{\frac{3 M_{p}^{2} \Omega_{\Lambda}}{3 n^{2} M_{p}^{2}+\gamma L^{-2} \ln \left(M_{p}^{2} L^{2}\right)+\beta L^{-2}}} \cos (\sqrt{|k|} y)\right] .
\end{aligned}
$$


The effective equations of state for dark energy and matter are defined by [32]

$$
\omega_{\Lambda}^{\mathrm{eff}}=\omega_{\Lambda}+\frac{\Gamma}{3 H}, \quad \omega_{m}^{\mathrm{eff}}=-\frac{1}{r_{m}} \frac{\Gamma}{3 H}
$$

Here $r_{m}=\rho_{m} / \rho_{\Lambda}$, and $\Gamma=Q / \rho_{\Lambda}=3 H\left(1+r_{m}\right)$, is the decay rate of dark energy into matter. Making use of (27) in (24) and (25), we have

$$
\begin{gathered}
\dot{\rho}_{\Lambda}+3 H\left(1+\omega_{\Lambda}^{\mathrm{eff}}\right) \rho_{\Lambda}=0 \\
\dot{\rho}_{m}+3 H\left(1+\omega_{m}^{\mathrm{eff}}\right) \rho_{m}=0 .
\end{gathered}
$$

We add a logarithmic correction term to the entropy

$$
S_{\Lambda}=S_{\Lambda}^{(0)}+S_{\Lambda}^{(1)}
$$

where

$$
S_{\Lambda}^{(1)}=-\frac{1}{2} \ln \left(C T^{2}\right)
$$

is the first order correction term to the entropy involving temperature $T$ and the heat capacity $C$. The later quantity is defined as

$$
C=T \frac{\partial S_{\Lambda}^{(0)}}{\partial T}
$$

To perform this analysis, we assume $\beta=0$. Now using (31), we have

$$
C=-\frac{8 \pi}{3}\left[3 n^{2} M_{p}^{2}\left(1+3 \omega_{\Lambda}^{0}\right)-\left(1-3 \omega_{\Lambda}^{0}\right) \gamma\left(L^{0}\right)^{-2} \ln \left(M_{p}^{2}\left(L^{0}\right)^{2}\right)+2 \gamma\left(L^{0}\right)^{-2}\right]\left(L^{0}\right)^{2} .
$$

For the case of the interacting ECHDE model, one obtains

$$
d S_{\Lambda}=8 \pi^{2} n^{2} M_{p}^{2}\left(1+3 \omega_{\Lambda}\right) L d L
$$

where

$$
\begin{aligned}
1+3 \omega_{\Lambda} & =\frac{1}{8 \pi^{2} n^{2} M_{p}^{2} L} \frac{d S_{\Lambda}}{d L} \\
& =\frac{1}{8 \pi^{2} n^{2} M_{p}^{2} L}\left[\frac{d S_{\Lambda}^{(0)}}{d L}+\frac{d S_{\Lambda}^{(1)}}{d L}\right]
\end{aligned}
$$


By comparing (26) and (35), we have

$$
\begin{aligned}
\frac{Q}{9 M_{p}^{2} \Omega_{\Lambda} H^{3}}= & -\frac{2}{3}+\frac{1}{3}\left(\frac{-2 \gamma L^{-2}+4 \gamma L^{-2} \ln \left(M_{p}^{2} L^{2}\right)+6 n^{2} M_{p}^{2}}{3 n^{2} M_{p}^{2}+\gamma L^{-2} \ln \left(M_{p}^{2} L^{2}\right)}\right) \\
& \times\left[1-\sqrt{\frac{3 M_{p}^{2} \Omega_{\Lambda}}{3 n^{2} M_{p}^{2}+\gamma L^{-2} \ln \left(M_{p}^{2} L^{2}\right)}} \cos (\sqrt{|k|} y)\right] \\
& -\frac{1}{9 n^{2} M_{p}^{2}}\left[3 n^{2} M_{p}^{2}\left(1+3 \omega_{\Lambda}\right)-\left(1-3 \omega_{\Lambda}\right) \gamma\left(L^{0}\right)^{-2} \ln \left(M_{p}^{2}\left(L^{0}\right)^{2}\right)+2 \gamma\left(L^{0}\right)^{-2}\right] \frac{L^{0}}{L} \frac{d L^{0}}{d L} \\
& -\frac{1}{24 \pi^{2} n^{2} M_{p}^{2} L} \frac{d S_{\Lambda}^{(1)}}{d L} .
\end{aligned}
$$

In this way we provide the relation between the interaction term of the dark components and the thermal fluctuation.

\section{CONCLUSION}

It is interesting to ask whether thermodynamics in an accelerating universe can reveal some properties of dark energy. It was first pointed out in [33] that the hyperbolic second order partial differential Einstein equation has a predisposition to the first law of thermodynamics. The profound connection between the thermodynamics and the gravitational field equations has also been observed in the cosmological situations [34 39]. This connection implies that the thermodynamical properties can help understand the dark energy, which gives strong motivation to study thermodynamics in the accelerating universe.

On the other side, in the absence of a symmetry that forbids the interaction between two dark components of the universe there is nothing, in principle, against it. Further, the interacting dark mater-dark energy (the latter in the form of a quintessence scalar field and the former as fermions whose mass depends on the scalar field) has been investigated at one quantum loop with the result that the coupling leaves the dark energy potential stable if the former is of exponential type but it renders it unstable otherwise. Thus, microphysics seems to allow enough room for the coupling; however, this point is not fully settled and should be further investigated. The difficulty lies, among other things, in that the very nature of both dark energy and dark matter remains unknown whence the detailed form of the coupling cannot be elucidated at this stage.

In this Paper, we investigated the model of interacting holographic dark energy with the inclusion of entropy corrections to the holographic dark energy. These corrections are 
motivated from the LQG which is one of the promising theories of quantum gravity. We provided a thermodynamical description of the ECHDE model in a universe with spacial curvature. We assumed that in the absence of a coupling, the two dark components remain in separate thermal equilibrium and that the presence of a small coupling between them can be described as stable fluctuations around equilibrium. Finally, resorting to the logarithmic correction to the equilibrium entropy we derived an expression for the interaction term in terms of a thermal fluctuation.

[1] A.G. Riess, et al., Astron. J. 116 (1998) 1009;

S. Perlmutter, et al., Astrophys. J. 517 (1999) 565;

S. Perlmutter, et al., Astrophys. J. 598 (2003) 102;

P. de Bernardis, et al., Nature 404 (2000) 955.

[2] T. Padmanabhan, Phys. Rep. 380 (2003) 235;

P. J. E. Peebles, B. Ratra, Rev. Mod. Phys. 75 (2003) 559;

E.J. Copeland, M. Sami, S. Tsujikawa, Int. J. Mod. Phys. D 15 (2006) 1753.

[3] A. Cohen, D. Kaplan, A. Nelson, Phys. Rev. Lett. 82 (1999) 4971.

[4] S. D. H. Hsu, Phys. Lett. B 594 (2004) 13.

[5] M. Li, Phys. Lett. B 603 (2004) 1.

[6] Q. G. Huang, M. Li, JCAP 0408 (2004) 013.

[7] E. Elizalde, S. Nojiri, S.D. Odintsov, P. Wang, Phys. Rev. D 71 (2005) 103504;

B. Guberina, R. Horvat, H. Stefancic, JCAP 0505 (2005) 001;

B. Guberina, R. Horvat, H. Nikolic, Phys. Lett. B 636 (2006) 80;

H. Li, Z. K. Guo, Y. Z. Zhang, Int. J. Mod. Phys. D 15 (2006) 869;

Q. G. Huang, Y. Gong, JCAP 0408 (2004) 006;

J. P. B. Almeida, J. G. Pereira, Phys. Lett. B 636 (2006) 75;

Y. Gong, Phys. Rev. D 70 (2004) 064029;

B. Wang, E. Abdalla, R. K. Su, Phys. Lett. B 611 (2005) 21.

[8] M. R. Setare, S. Shafei, JCAP 09 (2006) 011;

M. R. Setare, Phys. Lett. B 644 (2007) 99;

M. R. Setare, JCAP 0701 (2007) 023; 
M. R. Setare, Phys. Lett. B 654 (2007) 1;

M. R. Setare, Phys. Lett. B 642 (2006) 421.

[9] B. Wang, C. Y. Lin and E. Abdalla, Phys. Lett. B 637 (2005) 357;

M. R. Setare, Phys. Lett. B 642 (2006)1.

[10] G. t Hooft, gr-qc/9310026;

L. Susskind, J. Math. Phys. 36 (1995) 6377.

[11] X. Zhang, F. Q. Wu, Phys. Rev. D 72 (2005) 043524;

X. Zhang, F. Q. Wu, Phys. Rev. D 76 (2007) 023502;

Q. G. Huang, Y.G. Gong, JCAP 08 (2004) 006;

K. Enqvist, S. Hannestad, M. S. Sloth, JCAP 02 (2005) 004;

J. Y. Shen, B. Wang, E. Abdalla, R.K. Su, Phys. Lett. B 609 (2005) 200.

[12] D. Pavon, W. Zimdahl, Phys. Lett. B 628 (2005) 206.

[13] A. Sheykhi, Class. Quantum Grav. 27 (2010) 025007.

[14] T. Zhu and J-R. Ren, Eur. Phys. J. C 62 (2009) 413;

R-G. Cai et al, Class.Quant.Grav.26:155018,2009

[15] M. Jamil and M. U. Farooq, JCAP 03 (2010) 001;

R. Banerjee and B. R. Majhi, Phys. Lett. B 662 (2008) 62;

R. Banerjee and B. R. Majhi, JHEP 0806 (2008) 095;

B. R. Majhi, Phys. Rev. D 79 (2009) 044005;

R. Banerjee and S. K. Modak, JHEP 0905 (2009) 063;

S. K. Modak, Phys. Lett. B 671 (2009) 167.

[16] C. Rovelli, Phys. Rev. Lett. 77 (1996) 3288;

A. Ashtekar, J. Baez, A. Corichi, and K. Krasnov, Phys. Rev. Lett. 80 (1998) 904;

A. Ghosh and P. Mitra, Phys. Rev. D 71 (2005) 027502;

K.A. Meissner, Class. Quant. Grav. 21 (2004) 5245;

A.J.M. Medved and E.C. Vagenas, Phys. Rev. D 70 (2004) 124021

[17] H. Wei, Commun. Theor. Phys. 52 (2009) 743.

[18] J.D. Bekenstein, Nuovo Cim. Lett. 4 (1972) 737;

J.D. Bekenstein, Phys. Rev. D 7 (1973) 2333;

J.D. Bekenstein, Phys. Rev. D 9 (1974) 3292.

[19] J. M. Bardeen, S. W. Hawking and B. Carter, Comm. Math. Phys. 31 (1973) 161; 
S. W. Hawking, Commun. Math. Phys. 43 (1975) 199;

G. W. Gibbons and S. W. Hawking, Phys. Rev. D 15 (1977) 2738.

[20] T. Padmanabhan, Phys. Rept. 406 (2005) 49;

T. Padmanabhan, Gen. Relativ. Grav. 40 (2008) 529;

T. Padmanabhan, arXiv:0910.0839v2 [gr-qc];

T. Padmanabhan, arXiv:0911.5004v2 [gr-qc].

[21] P.C.W. Davies, Rept. Prog. Phys. 41 (1978) 1313;

P.C.W. Davies, Class. Quant. Grav. 4 (1987 ) L225;

T.M. Davies et al, Class. Quant. Grav. 20 (2003) 2753;

H. M. Sadjadi, Phys. Rev. D 73 (2006) 063525;

H. M. Sadjadi and M. Jamil, arXiv:1002.3588v1 [gr-qc];

M. Jamil, E. N. Saridakis and M. R. Setare, Phys. Rev. D 81 (2010) 023007;

M. Jamil, E. N. Saridakis and M. R. Setare, arXiv:1003.0876v1 [hep-th].

[22] R.G. Cai, L.M. Cao, Y.P. Hu, Class. Quantum. Grav. 26155018 (2009);

R. Li, J. R. Ren, D. F. Shi, Phys. Lett. B 670 (2009) 446.

[23] L. Amendola, Phys. Rev. D 60 (1999) 043501;

L. Amendola, Phys. Rev. D 62 (2000) 043511;

L. Amendola and C. Quercellini, Phys. Rev. D 68 (2003) 023514;

L. Amendola and D. Tocchini-Valentini, Phys. Rev. D 64 (2001) 043509.

[24] W. Zimdahl, D. Pavon, L.P. Chimento, Phys. Lett. B 521 (2001) 133;

W. Zimdahl and D. Pavon, Gen. Rel. Grav. 35 (2003) 413;

L. P. Chimento, A. S. Jakubi, D. Pavon and W. Zimdahl, Phys. Rev. D 67 (2003) 083513.

[25] B. Wang, Y. Gong and E. Abdalla, Phys. Lett. B 624 (2005) 141.

[26] A. Sheykhi, Phys Lett B 681 (2009) 205.

[27] B. Wang, J. Zang, Ch.Y. Lin, E. Abdalla, S. Micheletti, Nucl. Phys. B 778 (2007) 69.

[28] S. Das, P.S. Corasaniti, J. Khoury, Phys. Rev. D 73 (2006) 083509.

[29] L. Amendola, S. Tsujikawa, M. Sami, Phys. Lett. B 632 (2006) 155;

L. Amendola, C. Quercellini, Phys. Rev. D 68 (2003) 023514;

[30] L.P. Chimento, A.S. Jakubi, D. Pavon,W. Zimdahl, Phys. Rev. D 67 (2003) 083513;

S. del Campo, R. Herrera, D. Pavn, Phys. Rev. D 70 (2004) 043540.

[31] B. Wang, C.Y Lin, D. Pavon, E. Abdalla, Phys. Lett. B 662 (2008) 1; 
M.R. Setare, E.C. Vagenas, Phys. Lett. B 666 (2008) 111;

A. Sheykhi, M.R. Setare, arXiv:0912.1408.

[32] M. Jamil and M. A. Rashid, Eur. Phys. J. C 56 (2008) 429;

M. Jamil and M. A. Rashid, Eur. Phys. J. C 58 (2008) 111;

M. Jamil and M. A. Rashid, Eur. Phys. J. C 60 (2009) 141;

M. Jamil and F. Rahaman, Eur. Phys. J. C 64 (2009) 97;

M. Jamil, Int. J. Theor. Phys. 49 (2010) 144.

[33] T. Jacobson, Phys. Rev. Lett. 75, 1260 (1995).

[34] M. Akbar and R. G. Cai, Phys. Rev. D 75084003 (2007);

M. Akbar and R. G. Cai, Phys. Lett. B 648 (2007) 243;

M. Akbar and R. G. Cai, Phys. Lett. B 635 (2006) 7;

M. Akbar, Chin. Phys. Lett. 24 (2007) 1158;

M. Akbar, Int. J. Theor. Phys. 48 (2009) 2665.

[35] R. G. Cai and L. M. Cao, Phys.Rev. D 75, 064008 (2007).

[36] R. G. Cai and S. P. Kim, JHEP 0502, 050 (2005).

[37] B. Wang, E. Abdalla and R. K. Su, Phys.Lett. B 503, 394 (2001);

B. Wang, E. Abdalla and R. K. Su, Mod. Phys. Lett. A 17, 23 (2002);

R. G. Cai and Y. S. Myung, Phys. Rev. D 67, 124021 (2003).

[38] R. G. Cai and L. M. Cao, Nucl. Phys. B 785 (2007) 135.

[39] A. Sheykhi, B. Wang and R. G. Cai, Nucl. Phys. B 779 (2007)1;

A. Sheykhi, B. Wang and R. G. Cai, Phys. Rev. D 76 (2007) 023515;

A. Sheykhi, B. Wang, Phys. Lett. B 678 (2009) 434;

A. Sheykhi, JCAP 05 (2009) 019;

A. Sheykhi, B. Wang, Mod. Phys. Lett. A, in press, arXiv:0811.4477. 